\title{
Beta Contabilístico e risco sistemático associado a projetos de investimento de capital
}

\author{
Julio César Silva Costa \\ Graduando em Ciências Contábeis pela Universidade Federal do Maranhão - UFMA \\ Graduando em Administração pela Universidade Estadual do Maranhão - UEMA \\ E-mail: jcesarma@globo.com
}

\section{RESUMO}

No ambiente de negócios cada vez mais instável, o risco e a incerteza sempre estão presentes em maior ou menor grau na tomada de decisão e devem ser, portanto, merecer atenção. No que diz respeito, particularmente, às decisões de investimento, o risco representa essencialmente a volatibilidade dos retornos esperados de um investimento. Retornos esses definidos pela expectativa de recebimento dos fluxos de caixa de um investimento. Em se tratando da análise de investimentos de capital, a determinação do risco é essencial para a seleção de projetos a serem executados. Para tanto, são utilizados critérios qualitativos, ditos subjetivos, e quantitativos, fundamentados em modelos matemáticos e estatísticos, para se obter uma medida concreta da volatibilidade dos fluxos de caixa previstos do projeto ou do seu risco. Neste trabalho, o Beta Contabilístico apresenta-se como uma medida para se mensurar o risco inerente a projetos de investimento de capital, sintetizada por Richard A. Brealey e Stewart C. Myers para fins pedagógicos.

Palavras-chave: Investimento, projetos, risco, Beta Contabilístico.

\section{Accounting Beta and Systematic Risk associated to capital investment projects}

\section{ABSTRACT}

In an ever increasingly unstable business environment, risk and uncertainty are always present to a higher or lower degree in decision making, and must, therefore, be carefully considered. Concerning, particularly, decisions related to investment, the risk represents essentially the volatility of the expected profits of an investment. When it comes to the analysis of capital investments, the appraisal of risk is essential for the choosing of projects to be executed. In order to do so, we made use of qualitative, or subjective, as well as quantitative, criteria, based on mathematical and statistic models, in order to obtain a concrete measurement of the predicted volatility of project cash flows, as well as their risk. In the present study, Accounting Beta is used to measure the inherent risk in capital investment projects, as defined by Richard A. Brealey and Stewart C. Myers for didactic purposes.

Key Words: Investment, Projects, Risk, Accounting Beta. 


\section{INTRODUÇÃO}

Uma das maiores preocupações da Moderna Administração Financeira se refere à agregação de valor. Talvez a principal forma de agregar valor a uma organização seja através dos investimentos que essa decide realizar. A razão disso é que os investimentos de capital, além de refletirem os objetivos organizacionais, têm efeito significativo no curto e longo prazo sobre a situação econômica de uma organização. Por esse motivo, a integração entre as políticas de investimento e os planos de longo prazo é fundamental para o crescimento e aumento da competitividade organizacional.

A questão de investir se torna, nesse contexto, um dos fatores críticos para aumento de competitividade e, sobretudo, para agregação de valor nas organizações. Portanto, a questão de investir assume relevância no cotidiano dos diversos setores de uma economia. As empresas devotam considerável atenção à análise desses investimentos, de forma a obter, no futuro, um razoável retorno sobre os valores aplicados.

Como o retorno futuro está sempre sujeito a alguma incerteza, e esta incerteza deve, naturalmente, influenciar o retorno exigido pelo investidor, há que se considerar o grau de risco envolvido nas decisões de investir.

O presente artigo objetiva trazer uma contribuição para o estudo da análise de risco/retorno voltada às decisões de projetos de investimento de capital, por meio de uma proposta de utilização do Beta Contabilístico como indicador de risco conjuntural ou sistemático para esses investimentos.

\section{INCERTEZA X RISCO}

Uma das maiores preocupações da Moderna Administração Financeira se refere à agregação de valor. Talvez a principal forma de agregar valor a uma organização seja através dos investimentos que essa decide realizar. A razão disso é que os investimentos de capital, além de refletirem os objetivos organizacionais, têm efeito significativo no curto e longo prazo sobre a situação econômica de uma organização. 
Por esse motivo, a integração entre as políticas de investimento e os planos de longo prazo é fundamental para o crescimento e aumento da competitividade organizacional.

A questão de investir se torna, nesse contexto, um dos fatores críticos para aumento de competitividade e, sobretudo, para agregação de valor nas organizações. Portanto, a questão de investir assume relevância no cotidiano dos diversos setores de uma economia. As empresas devotam considerável atenção à análise desses investimentos, de forma a obter, no futuro, um razoável retorno sobre os valores aplicados.

Como o retorno futuro está sempre sujeito a alguma incerteza, e esta incerteza deve, naturalmente, influenciar o retorno exigido pelo investidor, há que se considerar o grau de risco envolvido nas decisões de investir.

O presente artigo objetiva trazer uma contribuição para o estudo da análise de risco/retorno voltada às decisões de projetos de investimento de capital, por meio de uma proposta de utilização do Beta Contabilístico como indicador de risco conjuntural ou sistemático para esses investimentos.

\begin{tabular}{|c|c|}
\hline GRAU DE CONHECIMENTO SOBRE O & CONDIÇÃO OU ESTADO \\
\hline As alternativas são conhecidas; & RISCO \\
São atribuídas probabilidades aos \\
resultados.
\end{tabular}

Quadro 1 - Condições ou Estados organizacionais em relação ao conhecimento sobre o futuro Fonte: Adaptado de Weston (1975, p. 68).

Analisando o quadro 1, podemos concluir que nas decisões que envolvem risco podem ser utilizados modelos matemáticos e estatísticos, uma vez que as probabilidades são de certa forma conhecidas ou podem ser atribuídas aos resultados alternativos. Já em condições de incerteza, as alternativas são conhecidas, porém não é possível determinar as probabilidades a serem atribuídas aos resultados alternativos 
ou aos seus valores. Na visão de Kassai et al., (2000, p. 10), o risco é “(...) uma incerteza que pode ser medida (...)", enquanto que a incerteza “(...) é um risco que não pode ser avaliado".

Robichek e Myers (1971, p. 94) são mais enfáticos, definindo risco como “(...) eventos aleatórios, cuja distribuição de probabilidade é conhecida". Já incerteza "(...) diz respeito a casos em que as distribuições não são conhecidas, não sendo, portanto, inteiramente significativas".

Securato (1996) acredita que o risco pode ser definido em termos de probabilidade. O risco como probabilidade, segundo esse autor, pode ser definido como grau de incerteza ou possibilidade de perda. Igualmente, o risco representa a probabilidade de ocorrência de um evento gerador da perda ou da incerteza. Admite-se, nesses termos, que os eventos podem assumir dois resultados quando se propõe atingir algum objetivo, a saber:

Sucesso: se acontecer o evento que nos interessa (atingir o objetivo). Fracasso: o evento que nos interessa não se realiza (não atingir o objetivo).

Sendo, ainda, $\mathrm{Xi}$ o conjunto de resultados, $\mathrm{S}$ o conjunto de sucessos e $\mathrm{F}$ o conjunto de fracassos:

$$
\begin{aligned}
& \mathrm{F} \times \mathrm{S}=\mathrm{Xi} \text { e } \mathrm{F} \times \mathrm{S}=\text { (eventos mutuamente exclusivos) O risco será dado por: } \\
& \text { Risco }=\mathrm{p}(\mathrm{F})
\end{aligned}
$$

Como $p(F)+p(S)=1$

Risco $=1-p(S)$

Onde:

$p(F)=$ Probabilidade de ocorrer o resultado fracasso; $p(S)=$ Probabilidade de ocorrer o resultado sucesso. 
Assim, como as decisões são votadas para o futuro, o risco e a incerteza se associam intimamente ao processo decisório das organizações. Podemos dizer, então, que à medida que o ambiente de negócios se torna mais complexo e globalizado as decisões relacionadas com o risco ganham mais importância, já que uma parte substancial dos riscos gerados por essa nova conjuntura pode ser coberta.

Deste modo, as decisões de aceitar ou rejeitar alternativas de investimento dependem não somente do retorno exigido pelos investidores, mas também do ajuste deste retorno ao risco. Além do risco, as decisões de investir podem variar em relação a outros fatores como: informações disponíveis e avaliação diferente por parte dos investidores da mesma informação. Isso pode originar distribuições de probabilidades subjetivas no tocante a coerência dos resultados possíveis e comportamento dos investidores frente ao risco (Robichek; Myers, 1971). Nesse artigo concentramos nossas atenções apenas na influência que a variável risco exerce nas decisões de investimento.

\section{RELAÇÃO RISCO/RETORNO E CAPITAL ASSET PRICING MODEL (CAPM)}

Até meados da década de 1950, não havia uma teoria que conseguisse estabelecer uma relação adequada do binômio risco e retorno. Em 1952, Harry Markowitz escreveu um artigo clássico que pode ser considerado a base da moderna filosofia da administração de portfólios. Foi Markowitz quem determinou a fronteira eficiente de ativos de risco a partir da premissa de selecionar portfólios que maximizassem a taxa de retorno para um dado nível de risco e minimizassem o nível de risco para uma dada taxa de retorno esperada (Silva, 2002). Posteriormente, as idéias de Markowitz foram aperfeiçoadas por William F. Sharp e outros financistas até que se tornasse o Modelo de Precificação de Ativos - Capital Asset Pricing Model (CAPM). Segundo esse modelo, a relação linear entre o risco/retorno e o risco total de um investimento pode ser decomposto em duas partes:

Risco Não-Sistemático - Unsystematic Risk (Não-Diversificável): é aquele que afeta um único ativo ou um pequeno grupo de ativos (Ross; Westerfield; Jordan, 2002). 
O Risco Não- Sistemático depende exclusivamente das características de cada investimento e é função dos itens que podem afetar o desempenho da organização (como, por exemplo: a estrutura de capital, o risco de gerenciamento e o mercado de atuação da empresa).

Risco Sistemático - Systematic Risk (Diversificável): é o que tem efeito amplo no mercado, influenciando, portanto, um grande número de organizações como, por exemplo: inflação, recessão econômica e taxa de juros (Brighan; Houston, 1999). Este risco é também conhecido como o risco de mercado e não pode ser eliminado pelo processo de diversificação.

Para Ross, Westerfield e Jordan (2002), como o risco não-sistemático pode ser eliminado pela diversificação, não pode existir recompensa por assumi-lo. Por sua vez, o retorno esperado de um investimento depende unicamente do risco sistemático desse investimento.

O CAPM estabelece que o prêmio pelo risco esperado de qualquer investimento deve variar na razão direta com o seu risco sistemático, que é medido pelo valor do seu coeficiente beta ( $\beta \mathrm{i})$. Assim, só deveriam ser aceitos investimentos cuja rentabilidade compensasse o beta. O $\beta$ i é encontrado a partir da covariância dos retornos de:

Se $1<\beta \mathrm{i} \leq 0$ : um coeficiente beta menor do que 1 e maior ou igual a 0 significa que quando a taxa de retorno do mercado se move para cima ou para baixo, a taxa de retorno exigida sobre determinado ativo tende a se mover no mesmo sentido, porém em menor magnitude. Por exemplo, se o retorno do mercado aumenta em 10\% para determinada ação com beta igual a 0.8 , o retorno exigido sobre esta ação aumentará em $8 \%$.

Se $\beta \mathrm{i}<0$ : neste raro caso um coeficiente beta menor do que zero significa que quando a taxa de retorno de mercado se move para cima ou para baixo, a taxa de retorno exigida sobre determinado ativo tende a se mover no sentido contrário (correlação negativa).

Nesse sentido, o retorno esperado de um investimento aumenta linearmente em função do seu beta. Esse retorno esperado é calculado pela equação abaixo: 
$E[R ̌ i]=R f+\beta i^{*}(E[R m]-R f)$

Sendo:

Rf: taxa de um ativo livre de risco;

$E$ [Ři]: o retorno esperado de um ativo $E[R m]$ : retorno esperado do mercado; (E [Rm] - Rf): prêmio por se assumir o risco;

$\beta i$ índice beta do investimento (risco sistemático).

Assim sendo, o retorno esperado de um investimento deve estar positivamente associado ao Beta (risco). Esta associação é representada graficamente no CAPM por uma linha ascendente denominada Security Market Line (SML).

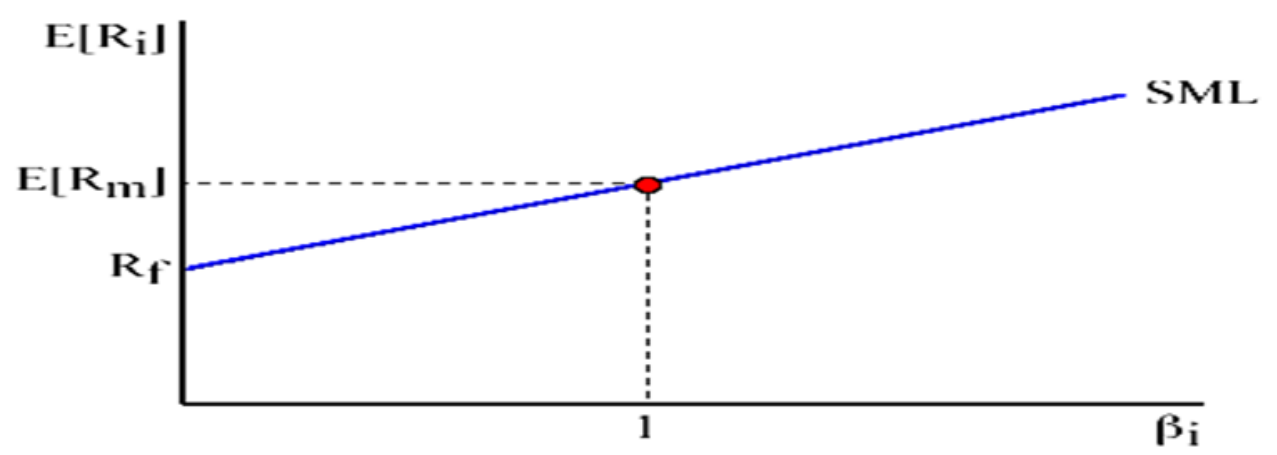

Figura 1 - Representação Gráfica da Security Market Line (SML)

Fonte: o autor

A inclinação da SML reflete o grau de aversão ao risco dos investidores. Quando não houver esse comportamento (aversão ao risco), não haverá prêmio pelo risco, de modo que a SML será horizontal $(\mathrm{Rm}=\mathrm{Rf})$. 
O CAPM se apresenta como um dos modelos mais amplamente aceitos na análise de investimentos. Contudo, esse modelo possui algumas restrições quanto ao seu uso, particularmente, na mensuração de risco de projetos, a saber:

Há dificuldades na sua utilização em projetos devido a diversos fatores envolvidos na mensuração de risco destes;

O CAPM é um modelo ex-ante (baseado em valores esperados), enquanto o cálculo do coeficiente beta tem por base dados históricos (ex-post);

Há dificuldades na sua utilização em empresas que atuam em diversos setores; e não pode ser utilizado em empresas que não possuem ações negociadas no mercado de capitais.

Outro aspecto interessante que deve ser destacado é o fato de que o índice beta (ou beta histórico) possui variações na sua forma de cálculo. Uma dessas variações é conhecida por Beta Contabilístico ou Contábil ( $\beta A c c)$. Os conceitos relativos ao Beta Contabilístico são mais bem elucidados nas próximas seções que tratam da aplicação desse índice na análise de risco em projetos.

\section{RELAÇÃO RISCO/RETORNO E AS DECISÕES DE INVESTIMENTO DE CAPITAL}

O risco no contexto financeiro, segundo Groppelli e Nikbakht (2000), representa uma medida de volatibilidade dos retornos associada à incerteza sobre resultados futuros. Retornos estes representados pelas expectativas de recebimentos ou fluxos de caixa previstos de qualquer investimento, conforme equação a seguir:

$$
\mathrm{E}\left[\sum_{\mathrm{R} i}\right]=\mathrm{R} 1 \mathrm{p} 1+\mathrm{R} 2 \mathrm{p} 2+\ldots+\mathrm{Rnpn}=i=1 \quad \mathrm{Ripi}
$$

Onde:

$E[\check{R i}]=$ Taxa de retorno

esperada; $\mathrm{Ri}$ = i-ésimo

retorno possível; 
pi $=$ Probabilidade do i-ésimo

retorno;

$\mathrm{n}$ = Número possível de

retornos.

Logo, o risco de um investimento está relacionado com a probabilidade de se obter um retorno menor do que o esperado, e quanto maior for a chance de baixos retornos ou retornos negativos, maior será o risco de um investimento.

Considerando os retornos em uma Distribuição Normal de Probabilidade (Distribuição Gaussiana), para determinar o risco podem ser utilizadas medidas costumeiras de dispersão, ou seja, Variância (๔2) ou Desvio Padrão (Ri).

Variância $[\check{R} i]=\sigma 2[$ Ři $]=\sum_{i=1}^{n}(R i-E[\check{R} i]) 2 p i$
Desvio Padrão $[$ Ři $]=$

Desvio Padrão $[\check{R i}]=\sqrt{\sigma}_{2}[\check{R} \mathrm{i}]=\sqrt{\sum^{n}}(\mathrm{Ri}-\mathrm{E}[\check{R} \mathrm{i}]) 2 \mathrm{pi}$

Coef $\operatorname{Var}=\sigma / E[R ̌ i]$

A relação entre risco e retorno é de tal importância que nenhum investimento deverá ser feito a menos que a taxa de retorno esperada seja suficientemente alta para compensar o investidor pelo risco percebido do investimento (Brigham; Houston, 1999).

Para Duarte Junior (1999), risco é um conceito multidimensional, que cobre quatro grandes grupos, a saber:

Risco de Mercado: depende do preço do ativo diante as condições de mercado. Pode ser dividido em risco do mercado acionário, risco do mercado de câmbio, risco do mercado de juros e risco do mercado de commodities.

Risco Operacional: está relacionado a possíveis perdas com resultados de 
sistemas e/ou controles inadequados, falhas de gerenciamento e erros humanos.

Risco de Crédito: representa o risco relacionado com possíveis perdas (recursos que não mais serão recebidos) quando um dos contratantes não honra os seus compromissos.

Risco Legal: está relacionado a possíveis perdas quando um contrato não pode ser legalmente amparado (por exemplo: documentação insuficiente, insolvência, ilegalidade, falta de representatividade e/ou autoridade por parte do negociador).

Em se tratando especificamente de projetos de investimentos de capital, são considerados relevantes os seguintes grupos de risco (Brigham; Houston, 1999):

Risco Isolado do Projeto: risco do investimento considerando como o único ativo da organização, ignorando a diversificação, tanto da empresa quanto dos seus acionistas. Geralmente é medido pelo desvio padrão $(\sigma)$ ou coeficiente de variação (desvio padrão dividido pela média) do Valor Presente Líquido ou da Taxa Interna de Retorno Mensurado pelo impacto do projeto nas incertezas sobre os lucros futuros.

Risco da Empresa: este risco reflete os efeitos do investimento nos resultados da organização, considerando a diversificação (outros investimentos da organização). Este risco depende do desvio padrão $(\sigma)$ do projeto e da correlação com o retorno de outros investimentos da organização.

Risco de Mercado (Risco Sistemático): reflete o efeito do investimento em uma carteira diversificada. Considera a diversificação e depende do desvio padrão $(\sigma)$ do projeto investimento de capital e da sua correlação com mercado acionário. É mensurado através do efeito do projeto no beta da empresa.

Os riscos de mercado (considerado o melhor) e corporativo são muito importantes nas decisões de investimentos de capital. Contudo, o risco isolado é o mais fácil de ser mensurado. Além disso, em projetos de investimento de capital de uma organização altamente correlacionados com os seus outros ativos e com a economia, geralmente o risco isolado reflete tanto o risco corporativo quanto o risco de mercado. 


\section{ANÁLISE DE RISCO EM PROJETOS DE INVESTIMENTO DE CAPITAL}

Após terem sido abordados alguns conceitos relativos ao risco e sua quantificação, discutiremos agora a análise de risco de projetos.

Segundo Samsão e Woiler (1996), algumas medidas indicam de que maneira é possível abordar de modo simplificado o problema do risco em projetos, estas são:

Estimativas cuidadosas: o processo de informações do projeto deve ser feito de tal maneira que as projeções sejam confiáveis;

Ajustes empíricos: a experiência passada deve ser observada de modo a não ser superestimada ou mesmo subestimada alguma informação. O ideal é serem elaboradas projeções de fatores mais relevantes em três níveis (pessimista, médio e otimista) e calcular o índice de análise para combinações destes níveis;

Ajuste da taxa de desconto ao risco: representa uma das formas de se ajustar o risco ao projeto (ajuste a priori). Geralmente, baixas taxas de desconto são aplicadas a projetos considerados menos arriscados e vice-versa.

Ajuste pela certeza equivalente: refere-se aos fatores que refletem a porcentagem de certa entrada de caixa que o responsável pela tomada de decisão aceitaria em troca das entradas de caixa esperadas (ajuste dos fluxos de caixa ao risco). O ajuste pela equivalência de certeza é feito através de um coeficiente de certeza equivalente ( $\alpha$ j) para cada período, da seguinte maneira:

Sendo o Valor Presente líquido de um projeto dado por:

$\mathrm{NPV}=\sum_{t=0}^{n} \mathrm{CFt} /(1+\mathrm{Ri}) \mathrm{t}$

(8)

Ajustando o coeficiente de certeza equivalente ( $\alpha \mathrm{j})$ aos fluxos de caixa de cada período: 
$\mathrm{NPV}=\sum_{t=0}^{n} \alpha j^{*} \mathrm{CFt} /(1+\mathrm{Ri}) \mathrm{t}$

Onde:

$0<\alpha j<1$ varia inversamente com o grau de risco associado a cada fluxo, ou seja, quanto maior o grau de risco associado a cada fluxo de caixa, menor é o coeficiente. Este coeficiente será determinado pela maior ou menor aversão ao risco por parte do investidor. Sendo:

$\alpha j=[$ Fluxo de caixa certo/Fluxo de caixa com risco $]$

Análise de sensibilidade: a análise de sensibilidade mede em que grau uma alteração prefixada de uma ou mais variáveis do projeto altera o resultado final. Nessa análise procura- se saber quais os elementos críticos na decisão de investimento.

Além dessas medidas existem critérios de avaliação de risco em projetos tanto quantitativos quanto qualitativos. Os critérios qualitativos são mais subjetivos e são geralmente utilizados quando há falta de informações para a análise (Kassai et al., 2000). As principais técnicas passíveis de serem utilizadas por uma análise baseada em critérios qualitativos são: pesquisa de mercado, técnica Delph, painel de especialistas, dramatização e criação de cenários, analogias históricas e de fenômenos naturais e análise de impactos cruzados.

Já os critérios quantitativos são fundamentados em modelos matemáticos e/ou estatísticos que procuram ajustar ou o fluxo de caixa ou a taxa de desconto do projeto ao risco. Dentre os critérios de análise quantitativa de risco destacam-se: análise de sensibilidade, análise de cenários, árvore de decisão, simulação de Monte Carlo, método de Hiller, Maximin, Maxmax, Hurwicz, Savage, Capital Asset Pricing Model (CAPM) e Arbitrage Pricing Theory (APT).

Não é objetivo deste estudo, dada a limitação em sua extensão, discutir os critérios (qualitativos e quantitativos) de análise de risco em projetos acima citados. $\mathrm{O}$ 
seu escopo é introduzir o Beta Contabilístico como um dos critérios de análise quantitativa de risco em projetos. Nesse sentido, nas próximas seções nos preocupamos em demonstrar como esse método pode ser aplicado na quantificação de risco de projetos.

\section{BETA CONTABOLÍSTICO: CONCEITOS E FORMAS DE MENSURAÇÃO}

A literatura financeira usualmente define Beta Contabilístico como uma medida análoga ao Beta de Mercado, simplesmente substituindo taxas de retorno de ações por medidas de retorno contábil e utilizando índices contábeis consolidados para todas as empresas como índice de mercado (Securato et al., (2004).

Beaver e Manegold (in Almisher e Kish, 2000), definem Beta Contabilístico de um ativo como sendo a covariância entre a série de retornos contábeis deste e a série de retornos de um índice econômico global, dividida pela variância dos retornos do índice econômico global.

Muitos estudos, conforme colocado por Brighan e Gapenski (1996), se destinaram a analisar a possível relação entre o Beta de Mercado (Beta Real) e o Beta Contabilístico. Apesar da diversidade de resultados a maior parte desses estudos, segundo os referidos autores, sustenta a conclusão de que as empresas com Beta Contabilístico elevado tendem a ter elevado Beta de Mercado, enquanto que as empresas com Beta Contabilístico baixo tendem a ter Beta de Mercado também baixo. Não obstante, as correlações são, geralmente, de apenas 0,5 a 0,6. Assim o Beta Contabilístico estabelece uma proxy do risco sistemático (risco conjuntural) de uma empresa.

O Beta Contabilístico ( $\beta A c c)$ é encontrado a partir da regressão entre o Retorno sobre Ativos da Empresa - ROAi (EBIT /Ativo) e o Retorno sobre Ativos Médio de uma grande amostra de empresas do mesmo setor ou de empresas semelhantes às negociadas em bolsa de valores (ROAMKT). Análogo ao cálculo do beta do modelo CAPM tradicional, o $\beta A c c$ é encontrado a partir da fórmula abaixo: 


$$
\beta A c c=\operatorname{Cov}(\text { ROAi, ROAMKT }) / \sigma 2(\text { ROAMKT })
$$

Ribeiro Neto e Fáma (2001), apoiados no estudo de Beaver e Manegold, propõem o cálculo do Beta Contabilístico a partir da razão entre a diferença da variação dos lucros da empresa ( $\Delta$ Lucros Empresa) e a taxa de juros de um ativo livre de risco (Rf) e a diferença entre a variação dos lucros do setor em que a empresa pertence ( $\Delta$ Lucros Setor) e esta mesma taxa livre de risco (Rf), conforme demonstrado na equação abaixo.

$$
\beta A c c=(\Delta \text { Lucros Empresa }- \text { Rf }) /(\Delta \text { Lucros Setor }- \text { Rf })
$$

Bowman (in Mendonça Neto; Matsumoto, 2004), demonstrou diversas associações teóricas entre diversas variáveis contábeis e o risco sistemático. Ele formulou uma associação entre o Beta Contabilístico e o Beta de Mercado, através da seguinte equação:

$$
\beta i=\beta \operatorname{Acc}(\mathrm{Sm} / \mathrm{Si})
$$

Onde:

$\beta i$ : Beta de mercado do ativo i;

$\beta$ Acc: Beta contabilístico do ativo;

Sm: Valor total da carteira composta por todos os ativos do mercado; Si: Valor de mercado do ativo i.

A principal vantagem da utilização do Beta Contabilístico como medida de risco conjuntural ou sistemático está no fato de que, ao contrário do Beta tradicional, que se aplica somente a empresas que negociam ações em bolsa de valores, este indicador pode ser utilizado tanto em empresas de capital aberto quanto em empresas de capital fechado ou mesmo micro e pequenas empresas.

Segundo Mendonça Neto e Matsumoto (2004), no caso brasileiro, onde muitas 
empresas são de capital fechado e não possuem ações negociadas em bolsa de valores, é impossível calcular o beta de um ativo a partir de informações históricas de seu retorno, visto que essas informações não existem. Desse modo, o Beta Contabilístico resolve o problema de falta de informações de mercado na aplicação do CAPM em empresas de capital fechado, micro e

Como o valor presente dos fluxos de caixa de um projeto é extremamente sensível às flutuações da receita, podemos relacionar esses fluxos de caixa com o valor presente da receita:

$\beta$ Projeto * [VP (projeto) /VP (Receita)] $=\beta$ Receita* [VP (Receita) /VP (Receita)] $\beta C F^{*}[$ VP (Custos Fixos)/VP (Receita)] - $\beta \mathrm{CV}^{*}[\mathrm{VP}$ (Custos Variáveis)/VP (Receita)] $\therefore$

$\beta$ Projeto * [VP (projeto) $/ \mathrm{VP}$ (Receita)] $=\beta$ Receita $-\beta C F^{*}[\mathrm{VP}$ (Custos Fixos) $/ \mathrm{VP}$ (Receita)] $\beta C V^{*}[\mathrm{VP}$ (Custos Variáveis)/VP (Receita)] $\therefore$

$\beta$ Receita $=\beta \mathrm{CF}^{*}[\mathrm{VP}$ (Custos Fixos) $/ \mathrm{VP}$ (Receita) $]+\beta C \mathrm{~V}^{*}[\mathrm{VP}$ (Custos Variáveis) $/ \mathrm{VP}$ (Receita)]+ $\beta$ Projeto * [VP (projeto) /VP (Receita)]

Onde:

$\beta C F: 0$, pois quem quer que receba o pagamento relativo aos custos fixos tem risco praticamente nulo;

$\beta$ Receita $\cong \beta C V$, já que estas variáveis reagem ao mesmo direcionador - o volume de atividade.

Substituindo os betas acima na Equação (15):

$\beta$ Receita $=\beta$ Receita * $[$ VP (Custos Variáveis) $/$ VP (Receita) $]+\beta$ Projeto * $[$ VP (projeto) $/$ VP (Receita)] $\therefore$

$\beta$ Projeto * $[$ VP (projeto) $/ \mathrm{VP}$ (Receita) $]=\beta$ Receita $-\beta$ Receita * $[\mathrm{VP}$ (Custos Variáveis) $/ \mathrm{VP}$ (Receita)] $\therefore$ 
$\beta$ Projeto * $[$ VP (projeto) $/ \mathrm{VP}$ (Receita) $]=\beta$ Receita * $[1-$ VP (Custos Variáveis) $/ \mathrm{VP}$ (Receita)] $\therefore$

$\beta$ Projeto *VP (projeto) $=\beta$ Receita * $[$ VP (Receita) - VP (Custos Variáveis) $]$ Sendo:

VP $($ projeto $)=$ VP $($ Receita $)-$ VP $($ Custos Fixos $)-$ VP (Custos Variáveis) $\therefore$

VP (projeto) + VP (Custos Fixos) $=$ VP (Receita) - VP (Custos Variáveis)

Substituindo na equação:

$$
\begin{aligned}
& \beta \text { Projeto * VP (projeto) }=\beta \text { Receita * }[\mathrm{VP} \text { (projeto) }+\mathrm{VP} \text { (Custos Fixos) }] \therefore \\
& \beta \text { Projeto }=\beta \text { Receita * }[\mathrm{VP} \text { (projeto) }+\mathrm{VP}(\text { Custos Fixos) }] / \mathrm{VP} \text { (projeto) } \therefore \\
& \beta \text { Projeto }=\beta \text { Receita * }[1+\mathrm{VP} \text { (Custos Fixos) / VP (projeto) }]
\end{aligned}
$$

Observamos que devido à volatibilidade das receitas previstas do projeto, medida pelo $\square$ Receita, o beta de um projeto acaba sendo proporcional à razão entre o valor presente dos custos fixos e o valor presente do projeto. Portanto, projetos cuja estrutura de custos é composta, em sua maior parte, de custos fixos, ou seja, projetos com alta alavancagem operacional, tendem a ter betas maiores (projetos mais voláteis).

Para demonstrar a aplicação de modelo acima apresentado, utilizamos um caso hipotético de uma da empresa Alfa que está analisando um dos seus projetos (Projeto Risk), que possui a seguinte previsão de fluxos de caixa: 
Tabela 1 - Fluxo de Caixa do Projeto Risk

\begin{tabular}{|c|c|c|c|c|}
\hline & & & & Em $\$ 1.00$ \\
\hline Ano & $\begin{array}{l}\text { Receitas } \\
\text { (a) }\end{array}$ & $\begin{array}{l}\text { Custos } \\
\text { Fixos } \\
\text { (b) }\end{array}$ & $\begin{array}{l}\text { Custos } \\
\text { Variáveis } \\
\text { (c) }\end{array}$ & $\begin{array}{l}\text { Fluxo de Caixa Líquido } \\
\qquad[a-(b+c)]\end{array}$ \\
\hline 0 & 0,00 & $150.000,00$ & 0,00 & $-150.000,00$ \\
\hline 1 & $75.000,00$ & $50.000,00$ & $15.000,00$ & $10.000,00$ \\
\hline 2 & $77.500,00$ & $50.000,00$ & $15.500,00$ & $12.000,00$ \\
\hline 3 & $80.000,00$ & $50.000,00$ & $16.000,00$ & $14.000,00$ \\
\hline 4 & $82.750,00$ & $50.000,00$ & $16.550,00$ & $16.200,00$ \\
\hline 5 & $85.000,00$ & $50.000,00$ & $17.000,00$ & $18.000,00$ \\
\hline 6 & $90.500,00$ & $50.000,00$ & $18.100,00$ & $22.400,00$ \\
\hline 7 & $92.000,00$ & $50.000,00$ & $18.400,00$ & $23.600,00$ \\
\hline 8 & $110.000,00$ & $50.000,00$ & $22.000,00$ & $38.000,00$ \\
\hline 9 & $116.500,00$ & $50.000,00$ & $23.300,00$ & $43.200,00$ \\
\hline 10 & $125.000,00$ & $50.000,00$ & $25.000,00$ & $50.000,00$ \\
\hline
\end{tabular}

Fonte: Elaborada pelo próprio autor

Sendo o custo de capital do projeto (não ajustado ao risco) igual a 5\% a.a.; o Valor Presente dos Custos Fixos e do Projeto são calculados assim:

$$
\begin{aligned}
& \text { VPProjeto }=-150.000+[10.000 /(1,05) 1+12.000 /(1,05) 2+14.000 /(1,05) 3+ \\
& 16.200 /(1,05) 4+18.000 /(1,05) 5+22.400 /(1,05) 6+23.600 /(1,05) 7+ \\
& 38.000 /(1,05) 8+43.200 /(1,05) 9+ \\
& 50.000 /(1,05) 10] \therefore
\end{aligned}
$$$$
\text { VPProjeto } \cong 27.683,11
$$$$
\text { VPCF }=150.000+50.000 *\{[1-1 /(1,05) 10] / 0,05\} \therefore
$$$$
\mathrm{VPCF} \cong 536.086,75
$$

Considerando que o beta da receita possa ser estimado tomando-se com base a correlação entre a receita de vendas da empresa e a receita de vendas do setor ao qual ela pertence, tendo como referência um período de 10 anos, tem-se o exposto na tabela abaixo: 
Tabela 2 - Relação entre a Receita de Vendas da Empresa Alfa e a Receita de Vendas do Setor

\begin{tabular}{|c|c|c|}
\hline Ano & $\begin{array}{c}\text { Receita de Vendas } \\
\text { da Empresa }\end{array}$ & $\begin{array}{c}\text { Receita de Vendas } \\
\text { do Setor }\end{array}$ \\
\hline 1 & $215.000,00$ & $3.656 .480,00$ \\
\hline 2 & $275.600,00$ & $4.251 .800,00$ \\
\hline 3 & $289.000,00$ & $6.245 .100,00$ \\
\hline 4 & $265.000,00$ & $6.548 .700,00$ \\
\hline 5 & $236.000,00$ & $7.451 .200,00$ \\
\hline 6 & $272.000,00$ & $6.954 .800,00$ \\
\hline 7 & $294.500,00$ & $8.156 .000,00$ \\
\hline 8 & $266.200,00$ & $7.845 .600,00$ \\
\hline 9 & $251.600,00$ & $7.801 .000,00$ \\
\hline 10 & $271.250,00$ & $7.985 .450,00$ \\
\hline
\end{tabular}

Fonte: Elaborada pelo próprio autor

Para realizar a análise de regressão linear entre a variável receita de venda da empresa Alfa (variável dependente - Y) e a variável receita de venda do mercado (variável independente - X) utilizamos as seguintes equações:

$$
\begin{aligned}
& \Sigma Y=n a+b(\Sigma X) \\
& \Sigma X Y=a(\Sigma X)+b(\Sigma X 2)
\end{aligned}
$$

Onde:

n: representa o número de pares de observações (10 no total);

a e b: representam os valores para a equação $Y=a+b X$, sendo b o Beta da Receita.

Para simplificar-se o cálculo de a e b, utilizamos as seguintes fórmulas, descritas por Stevenson (1981):

$$
\begin{aligned}
& \mathrm{b}=[\mathrm{n}(\Sigma \mathrm{XY})-\Sigma(\mathrm{X})(\Sigma \mathrm{Y})] /[\mathrm{n}(\Sigma \mathrm{X} 2)-(\Sigma \mathrm{X}) 2] \\
& \mathrm{a}=[(\Sigma \mathrm{Y})-\mathrm{b}(\Sigma \mathrm{X})] / \mathrm{n}
\end{aligned}
$$


Os valores de $\Sigma Y, \Sigma X, \Sigma X Y$ e $\Sigma$ X2 são calculados na tabela abaixo:

Tabela 3 - Análise de Regressão da Receita de Vendas da Empresa Alfa

\begin{tabular}{|c|c|c|c|c|}
\hline $\mathbf{n}$ & $\mathbf{Y}$ & $\mathbf{X}$ & $\mathbf{X}$ & $\mathbf{X Y}$ \\
\hline 1 & $215.000,00$ & $3.656 .480,00$ & $13.369 .845 .990 .400,00$ & $786.143 .200 .000,00$ \\
\hline 2 & $275.600,00$ & $4.251 .800,00$ & $18.077 .803 .240 .000,00$ & $1.171 .796 .080 .000,00$ \\
\hline 3 & $289.000,00$ & $6.245 .100,00$ & $39.001 .274 .010 .000,00$ & $1.804 .833 .900 .000,00$ \\
\hline 4 & $265.000,00$ & $6.548 .700,00$ & $42.885 .471 .690 .000,00$ & $1.735 .405 .500 .000,00$ \\
\hline 5 & $236.000,00$ & $7.451 .200,00$ & $55.520 .381 .440 .000,00$ & $1.758 .483 .200 .000,00$ \\
\hline 6 & $272.000,00$ & $6.954 .800,00$ & $48.369 .243 .040 .000,00$ & $1.891 .705 .600 .000,00$ \\
\hline 7 & $294.500,00$ & $8.156 .000,00$ & $66.520 .336 .000 .000,00$ & $2.401 .942 .000 .000,00$ \\
\hline 8 & $266.200,00$ & $7.845 .600,00$ & $61.553 .439 .360 .000,00$ & $2.088 .498 .720 .000,00$ \\
\hline 9 & $251.600,00$ & $7.801 .000,00$ & $60.855 .601 .000 .000,00$ & $1.962 .731 .600 .000,00$ \\
\hline 10 & $271.250,00$ & $7.985 .450,00$ & $63.767 .411 .702 .500,00$ & $2.166 .053 .312 .500,00$ \\
\hline$\Sigma$ & $\mathbf{2 . 6 3 6 . 1 5 0 , 0 0}$ & $\mathbf{6 6 . 8 9 6 . 1 3 0 , 0 0}$ & $\mathbf{4 6 9 . 9 2 0 . 8 0 7 . 4 7 2 . 9 0 0 , 0 0}$ & $\mathbf{1 7 . 7 6 7 . 5 9 3 . 1 1 2 . 5 0 0 , 0 0}$ \\
\hline
\end{tabular}

Fonte: Elaborada pelo próprio autor

Substituindo os valores, encontramos os coeficientes

$\mathrm{a}$ e b: $\mathrm{a}=223.984,6689$

$b=0,005924159$

A equação será então igual a: $Y=223.984,6689+0,005924159 X$, ou:

Receita de Vendas da Empresa $=223.984,6689+(0,005924159 \times$ Receita do Setor $)$

$\mathrm{b}=\beta$ Receita $=0,005924159$ mede a sensibilidade das vendas da empresa em relação ao setor da economia ao qual ela atua. O beta da receita menor que 1 significa que a empresa possui uma baixa correlação com as flutuações na receita do setor, ou seja, quando a demanda do setor tender a cair ou subir, as receitas de venda da 
empresa também tenderão a se moverem no mesmo sentido, porém em uma menor intensidade.

Utilizamos a Equação (16) para calcular o beta do Projeto Risk:

$\beta$ Projeto $=\beta$ Receita * $[1+\mathrm{VP}$ (Custos Fixos) $/ \mathrm{VP}$ (projeto) $]=0,005924159{ }^{*}[1$ $+536.086,75 / 27.683,11]$

$\beta$ Projeto $=0,1206$

Através do resultado encontrado pelo cálculo do beta do projeto, chegamos as seguintes constatações:

Calculada a volatibilidade das receitas (medida pelo beta da receita), o beta do projeto fica sendo proporcional ao índice do valor presente dos custos fixos sobre o valor presente do projeto (19,3651 - no caso do Projeto Risk). Assim, permanecendo os demais fatores constantes, quanto maior for esse índice (VP (Custos Fixos) / VP (projeto), maior será o beta (risco) do projeto e vice-versa.

Alavancagem operacional dos projetos representa uma medida de risco, pois projetos com uma estrutura de custos composta em sua maior parte de custos fixos são mais voláteis em relação às flutuações da demanda.

O beta do projeto mede a sensibilidade dos fluxos de caixa previstos do projeto em relação à conjuntura do mercado.

O beta do projeto pode ser utilizado como parâmetro de comparação com o beta da empresa (Beta de Mercado ou Beta Contabilístico) ou de outros investimentos para se avaliar o grau de risco (alto risco, baixo risco ou risco moderado) de um projeto.

\section{CONSIDERAÇÕES FINAIS}

O propósito deste artigo foi apresentar o Beta Contabilístico como uma medida de risco de projetos de investimento de capital. Para tanto, verificamos a existência de diversos modelos de tratamento de risco em projetos de investimento, dos mais 
subjetivos ditos qualitativos, aos que utilizam de modelos matemáticos e estatísticos.

Evidenciamos a pouca utilização de critérios que usam medidas contábeis para se quantificar a variável risco em projetos de investimento de capital. A explicação para esse fenômeno pode residir na dificuldade de se conciliar a utilização de informações passadas, onde são extraídas as medidas contábeis, com as predições sobre o futuro, horizonte de tempo ao qual se baseia a análise econômico-financeira de projetos de investimento de capital.

Diante do exposto, este artigo buscou conciliar a análise sobre eventos futuros de um projeto (fluxos de caixa previstos) com informações baseadas no que ocorreu (medidas contábeis), para se quantificar o risco de projetos através do Beta Contabilístico. Para esse fim, adotamos como modelo de Beta Contabilístico o proposto pelos professores de Finanças Richad A. Brealey e Stewart C. Myers.

Demonstramos através de um caso hipotético a aplicabilidade desse modelo, o que elucidou ainda mais o processo de se quantificar o risco de projetos pelo Beta Contabilístico.

Finalizando, esperamos que o presente artigo contribua para os estudos no campo das Finanças Corporativas, e mais especificamente para a análise de risco de projetos de investimentos de capital, à medida que buscou adaptar um instrumento utilizado na mensuração do risco sistemático total de empresas na mensuração do risco de projetos.

Em razão da natureza inovadora do estudo aqui apresentado, admite-se a possibilidade de aperfeiçoamento e aplicação do modelo.

\section{REFERÊNCIAS}

ALMISHER, M.A. e KISH, R. 2000. Accounting beta: anex anti proxy for risk within the ipo market. Journal of Financial and Strategic Decisions, 13(3):23-34.

BREALEY, R.A. e MYERS, S.C. 2006. Investimento de capital e avaliação. São Paulo, Bookman, 552 p. BRIGHAM, E.F. e GAPENSKI, L.C. 1996. Intermediate Financial Management. $5^{\mathrm{a}}$ ed., Orlando, DrydenPress, $940 \mathrm{p}$. 
e HOUSTON, J.F. 1999. Fundamentos da moderna Administração

Financeira. Rio de Janeiro, Campus. 736 p.

DUARTE JUNIOR, A.M. 1999. Risco: definições, tipos, medição e recomendações para seu gerenciamento. In: I Seminário Nacional de Risco Financeiro. João Pessoa. 1999. Anais... João Pessoa, PPGA-UFPB. p. 25-33.

FRANCIS, J.C. 1980. Investments: analysis and management. 3a ed., New York, McGraw-Hill, 672 p. GROPPELLI, A.A. e NIKBAKHT, E. 2000. Finance. 4를 ed., New York, Barron's, $270 \mathrm{p}$.

HAUGE, R.A. 2001. Modern investment theory. $5^{\mathrm{a}}$ ed., New Jersey, Prentice Hall, 656 p.

KASSAI, J.R.; et al., 2000. Retorno de investimento: abordagem matemática e contábil do lucro empresarial. $2^{\mathrm{a}}$ ed. São Paulo, Atlas, 256 p.

MENDONÇA NETO, J.N. e MATSUMOTO, A.S. 2004. A viabilidade do beta contábil para a estimativa do risco sistemático. In: $1^{\circ}$ Encontro Norte Nordeste de Finanças. Recife, 2004. Anais... Recife, 2004.

NAKAMURA, W.T. e MATIAS, F. 2005. Estudo empírico sobre metodologias alternativas de aplicação do CAPM no mercado de ações brasileiro. Universidade Presbiteriana Mackenzie, 02/2005. Disponível em: http://www.congressoeac.locaweb.com.br, acesso em:05/11/2006.

RIBEIRO NETO, R.M. e FAMÁ, R. 2001. Beta contabilístico: uma aplicação no mercado financeiro brasileiro. In: V SEMEAD FEA USP, São Paulo. Anais eletrônicos... São Paulo: FEA USP, 2001. Disponível em: www.labfin.com.br, acesso em: 20/12/2005.

ROBICHEK, A.A. e MYERS, S.C. 1971. Otimização das decisões financeiras. São Paulo, Atlas, $340 \mathrm{p}$.

ROSS, S.A., WESTERFIELD, R.W. e JORDAN, B.D. 2002. Princípios de Administração Financeira. $2^{a}$ ed. São Paulo, Atlas, 530 p.

SAMSÃO, W. e WOILER, F.M. 1996. Projetos: planejamento, elaboração e análise. São Paulo, Atlas, 296 p.

SECURATO, J.R. 1996. Decisões financeiras em condições de risco. São Paulo, Atlas, $244 \mathrm{p}$.

et al., Análise da relação entre indicadores contábeis e betas de mercado das empresas brasileiras negociadas na Bolsa de Valores de São Paulo no 
período 1995 - 2003. São Paulo, out. 2004. Disponível em: <http://www.ead.fea.usp.br, acesso em:20/06/2006.

SILVA, M.A.V.R. 2002. A utilização do índice beta do Modelo de Apreçamento de Ativos (CAPM) como medida de risco sistemático: uma análise do mercado acionário brasileiro. Revista Humanas - UNIFEI, 19:31-35.

STEVENSON, W.J. 1981. Estatística aplicada à Administração. São Paulo, Harper e Row. 495 p.

WESTON, J.F. 1975. Finanças de empresas: campo e metodologia. 5a., São Paulo, Atlas, $915 \mathrm{p}$.

Data de Submissão: 29/04/2007

Data de Aceite: 27/08/2007 